OPEN ACCESS

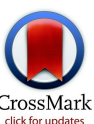

${ }^{1}$ Renal Division, Brigham and Women's Hospital, 75 Francis

Street, Boston, MA 02115, USA

${ }^{2}$ Channing Division of Network

Medicine, Department of

Medicine, Brigham and

Women's Hospital and Harvard

Medical School, Boston, USA

${ }^{3}$ Departments of Nutrition and

Epidemiology, Harvard T H Chan

School of Public Health, Boston,

USA

Correspondence to:

L Borgi lborgi@partners.org

Cite this as: BMJ 2016;353:i2351

http://dx.doi.org/10.1136/bmj.i2351

Accepted: 12 April 2016

\title{
Potato intake and incidence of hypertension: results from three prospective US cohort studies
}

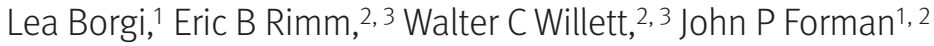

\section{ABSTRACT}

OBJECTIVE

To determine whether higher intake of baked or boiled potatoes, French fries, or potato chips is associated with incidence of hypertension.

DESIGN

Prospective longitudinal cohort studies.

SETTING

Healthcare providers in the United States.

\section{PARTICIPANTS}

62175 women in Nurses' Health Study, 88475 women in Nurses' Health Study II, and 36803 men in Health

Professionals Follow-up Study who were non-

hypertensive at baseline.

\section{MAIN OUTCOME MEASURE}

Incident cases of hypertension (self reported diagnosis by healthcare provider).

\section{RESULTS}

Compared with consumption of less than one serving a month, the random effects pooled hazard ratios for four or more servings a week were 1.11 (95\% confidence interval 0.96 to 1.28; P for trend=0.05) for baked, boiled, or mashed potatoes, 1.17 (1.07 to 1.27; P for trend $=0.001$ ) for French fries, and 0.97 (0.87 to 1.08; $P$ for trend=0.98) for potato chips. In substitution analyses, replacing one serving a day of baked, boiled, or mashed potatoes with one serving a day of nonstarchy vegetables was associated with decreased risk of hypertension (hazard ratio $0.93,0.89$ to 0.96 ).

CONCLUSION

Higher intake of baked, boiled, or mashed potatoes and French fries was independently and prospectively associated with an increased risk of developing hypertension in three large cohorts of adult men and women.

\section{Introduction}

Government agencies in the United States have established several programs in the past decade to provide healthy meals to children and low income populations, ${ }^{12}$ including the Healthy, Hunger-Free Act of 2010 (a school

\section{WHAT IS ALREADY KNOWN ON THIS TOPIC}

Because potatoes are high in both glycemic carbohydrates and potassium, their effect on risk of hypertension is unclear

The association of intake of white potatoes with hypertension has not been studied

\section{WHAT THIS STUDY ADDS}

Higher intakes of boiled, baked, or mashed potatoes, and French fries were associated with an increased risk of hypertension in adult women and men

Replacing one serving a day of boiled, baked, or mashed potatoes with one serving of a non-starchy vegetable was associated with a lower risk of developing hypertension lunch program) and the Special Supplemental Nutrition Program for Women, Infants, and Children (WIC). ${ }^{3}$ The Healthy, Hunger-Free Act initially restricted the provision of starchy vegetables to one cup a week; in 2009 the WIC program offered a monthly fruit and vegetable cash value voucher that excluded white potatoes. ${ }^{4}$ However, opposition from several state and local school lunch operators led to a removal of the restriction on starchy vegetables (including French fries) in school lunches in 2012,5 and, on the basis of an Institute of Medicine report requested by Congress, ${ }^{6}$ the restriction on white potatoes in the WIC fruit and vegetable cash voucher was lifted in 2015. The rationale for removing these limitations on potatoes included their high potassium content and insufficient evidence of their health effects. ${ }^{6}$ On the international front, the World Health Organization does not include potatoes as vegetables. ${ }^{7}$

Although potassium supplementation may be beneficial for chronic disease prevention, in particular prevention of hypertension, ${ }^{8}$ a six week feeding trial of 164 pre-hypertensive and hypertensive people found that diets rich in protein or monounsaturated fat reduced blood pressure compared with diets rich in carbohydrates. ${ }^{9}$ Because potatoes are high in both glycemic carbohydrates and potassium, their effect on risk of hypertension is unclear. The long term association of high potato intake with the risk of developing hypertension has not been examined. We therefore examined the prospective, independent association of consumption of baked, boiled, or mashed potatoes, French fries, and potato chips with incident hypertension in three large cohort studies that included 187453 participants with more than 20 years of follow-up.

\section{Methods}

\section{Study population}

We used data from the Nurses' Health Study (NHS; $\mathrm{n}=121700$ women, aged 30-55 at the time of cohort inception in 1976), the Nurses' Health Study II (NHS II; $\mathrm{n}=116430$ women, aged 25-42 at cohort inception in 1989), and the Health Professionals Follow-up Study (HPFS; $n=51529$ men, aged 40-75 at cohort inception in 1986). Participants returned a questionnaire every two years with health status updates, including information about hypertension diagnosed by a healthcare provider. Also, participants of NHS answered an extensive semiquantitative food frequency questionnaire in 1984, 1986, and then every four years thereafter; participants of NHS II and HPFS answered similar questionnaires every four years starting in 1991 (in NHS II) and 1986 (HPFS). The validity and reproducibility of these food frequency questionnaires, which ascertain intake of more than 130 foods and beverages, has been detailed previously. ${ }^{11} 11$ After exclusion of participants who did not provide dietary 
information and those who reported a diagnosis of hypertension at the baseline food frequency questionnaire (1984, 1991, and 1986 for NHS, NHS II, and HPFS, respectively), the study population consisted of 62175 women in NHS, 88475 women in NHS II, and 36803 men in HPFS.

\section{Assessment of hypertension}

A diagnosis of hypertension by a health professional was self reported on the baseline and biennial questionnaires. Studies in the three cohorts were conducted to validate this method of diagnosis of hypertension..$^{12-14}$ In NHS, $77 \%$ (39/51 cases) of self reported hypertension had a blood pressure above 160/95 mm $\mathrm{Hg}$ and a 100\% had a pressure above 140/90 mm Hg; none of the women who did not report a diagnosis of hypertension were found to be hypertensive by medical record review. In HPFS, of 114 participants without a self report of hypertension, only two were found to have hypertension. ${ }^{14}$ In a review of randomly chosen participants who self reported a diagnosis of hypertension in NHS II and HPFS, 94\% and $100 \%$ (39 participants) were confirmed to have hypertension. ${ }^{12-14}$ As this analysis was prospective, we excluded participants with self reported hypertension at the baseline questionnaire from our study. Incident cases of hypertension were those who reported a diagnosis of hypertension on subsequent questionnaires with a date of diagnosis that was after the baseline questionnaire.

\section{Assessment of potato intake}

In 1984, 1991, and 1986 a detailed food frequency questionnaire was sent to all participants in NHS, NHS II, and HPFS, respectively. Approximately every four years thereafter, similar questionnaires were sent out to update habitual dietary intake. Participants answered how often, on average over the previous year, they had consumed each food in a standard portion size. Three questions asked about potatoes: baked, boiled, or mashed potatoes (either one potato or one cup), ascertained as a single question; French fries ( $4 \mathrm{oz}$ or one serving); and potato or corn chips (small bag or $1 \mathrm{oz}$ ). Nine different response categories could be selected, ranging from "never or less than once a month" to "six or more a day." Because the number of people in some of these nine categories was low (for example, consuming a food six times a day was a rare event), we collapsed the smaller categories into larger ones out of necessity. As a result, three potato variables-French fries, potato chips, and the combination of baked, boiled, or mashed potatoes (which were ascertained as a group on the questionnaire with a single question)-were collapsed into four categories for the purpose of analysis. When we analyzed baked, boiled, or mashed potatoes together with French fries, the number of participants in each response category was larger, permitting us to examine a broader range of intake (five rather than four categories). In previous validation studies, the deattenuated Pearson correlations between potato intake reported on food frequency questionnaires and on multiple one week dietary records (considered the gold standard) were 0.69 for baked, boiled or mashed potatoes, 0.56 for French fries, and 0.55 for potato chips. ${ }^{1516}$

\section{Assessment of covariates}

Updated information about weight, smoking status, physical activity (estimated as metabolic equivalent tasks (METs)) was obtained on biennial questionnaires. Body mass index was calculated as weight (in $\mathrm{kg}$ ) divided by height (in $\mathrm{m}$ ) squared. Potential dietary confounders were also ascertained on the food frequency questionnaires, such as alcohol, whole grains, total caloric intake, total whole fruit and vegetable intake, and consumption of sugar sweetened and artificially sweetened drinks and animal flesh (red and processed meat, poultry, and fish). These covariates have been validated with correlation coefficients of, for example, 0.97 for weight, 0.79 for physical activity, and 0.90 for alcohol. ${ }^{118}$ Race/ethnicity was self reported.

\section{Statistical analysis}

As the month and year of the diagnosis of hypertension by a health professional was provided by participants, we used an interval censoring model by calculating each participant's person time (in months) from the date of the baseline questionnaire to the date of diagnosis of hypertension, date of death, or end of follow-up (2010 for NHS and HPFS, 2011 for NHS II), whichever came first.

We used a cumulative average of a participant's intake to decrease within person variation. We grouped consumption of baked, boiled or mashed potatoes, French fries, and potato chips into four categories, from " $\leq 1$ serving/month" to " $\geq 4$ servings/week." We also analyzed a variable that combined intake of baked, boiled, or mashed potatoes and French fries, which we grouped into five categories: " $<1$ serving/month," "1-3 servings/month," "1-3 servings/week," "4-6 servings/ week," and " $\geq 1$ serving/day."

We then used Cox proportional hazards regression to calculate the hazard ratios and 95\% confidence intervals for incident hypertension, using “ $<1$ serving/month" as the reference group for all analyses. We adjusted for the following potential confounders, updating them as time varying covariates by using information from successive questionnaires: age; body mass index; change in weight; race/ethnicity (white, African-American, Asian, Hispanic, other); family history of hypertension; smoking status (current/past); physical activity (METs/week); postmenopausal status; oral contraceptive use (in NHS II); use of non-narcotic analgesics (non-steroidal anti-inflammatory drugs, acetaminophen (paracetamol), and aspirin); total energy intake (kcal/day); and intakes of alcohol (g/day), total whole fruits and total vegetables, animal flesh, whole grains, and sugar sweetened and artificially sweetened drinks. We used fixed effects meta-analysis to pool adjusted multivariable hazard ratios for the three cohorts. We then calculated the attributable fraction of hypertension in NHS II (the cohort closest in age to participants in the WIC program) by using the following formula: $\mathrm{AF} \%=(\mathrm{HR}-1 / \mathrm{HR}) p d \times 100$, where $p d$ is the proportion of cases exposed the risk factor, which here represents potato consumption. ${ }^{19}$

We then used substitution analyses to determine whether replacing one serving a day of baked, boiled, or mashed potatoes with other starchy vegetables (peas, 
lima beans, corn, and sweet potatoes), non-starchy vegetables, or French fries, was associated with an increased or decreased risk of developing hypertension. In these analyses, the hazard ratio for starchy vegetables, non-starchy vegetables, or French fries represents the "effect" of replacing one serving a day of baked, boiled, or mashed potatoes with one of these foods.

We then did several secondary analyses. Firstly, we added intake of micronutrients (potassium, calcium, magnesium, sodium, and fiber) to our multivariable models. Secondly, we repeated our analyses excluding body mass index and weight changes from our models. Thirdly, we introduced trans fat and saturated fat into our multivariable models; polyunsaturated fat was added only into the multivariable model analyzing potato chips and hypertension. Fourthly, we created a stratified model and introduced multiplicative interaction terms to our unstratified multivariable models to analyze whether the associations of potato intake with hypertension varied significantly according to age and body mass index. Fifthly, we repeated our analyses without cumulatively averaging participants' potato intake (that is, using simple updating). Finally, we reanalyzed the pooled hazard ratios by using random effect models.

We used SAS software (version 9.4) for all analyses. All $P$ values were two sided.

\section{Patient involvement}

No patients were involved in setting the research question or the outcome measures, nor were they involved in developing plans for recruitment, design, or implementation of the study. No patients were asked to advise on interpretation or writing up of results. There are no plans to disseminate the results of the research to study participants or the relevant patient community.

\section{Results}

During 2938961 person years of follow-up, 77726 participants reported a diagnosis of hypertension (35728 cases/1034257 person years in NHS, 25246 cases/1344475 person years in NHS II, and 16752 cases/560229 person years in HPFS). Table 1 reports baseline characteristics for each cohort according to the lowest and highest intake category of baked, boiled, or mashed potatoes, French fries, and potato chips. Participants who consumed four or more servings a week of any type of potatoes were, in general, less physically active and smoked more than participants whose consumption was less than one serving a week.

In pooled analyses, participants who consumed at least one serving a day of either baked, boiled, or mashed potatoes or French fries had an independently increased risk of incident hypertension compared with those who ate less than one serving a month of these foods, with a multivariable pooled hazard ratio of 1.13 (95\% confidence interval 0.91 to 1.40; $P$ for trend $=0.02$ ) in a random effect model and 1.12 (1.02 to 1.24; P for trend <0.001) in a fixed effect model (P for heterogeneity <0.001) (table 2). The multivariable hazard ratios for these comparisons in NHS, NHS II, and HPFS were 1.07 (0.92 to 1.24), 1.42 (1.18 to 1.72), and 0.96 (0.79 to 1.16), respectively.

Baked, boiled, or mashed potatoes were associated with an increased adjusted risk of developing hypertension when we compared intake of four or more servings a week with less than one serving a month in NHS (hazard ratio $1.13,1.02$ to 1.26 ; P for trend=0.01) and NHS II

\begin{tabular}{|c|c|c|c|c|c|c|}
\hline \multirow[b]{2}{*}{ Characteristic } & \multicolumn{2}{|c|}{ Baked, boiled, or mashed potatoes } & \multicolumn{2}{|l|}{ French fries } & \multicolumn{2}{|l|}{ Potato chips } \\
\hline & $<1$ per month & $\geq 4$ per week & $<1$ per month & $\geq 4$ per week & $<1$ per month & $\geq 4$ per week \\
\hline \multicolumn{7}{|l|}{ Nurses' Health Study } \\
\hline Age, years & $51(45-57)$ & $51(45-57)$ & $52(47-58)$ & $46(41-53)$ & $53(47-58)$ & $45(41-51)$ \\
\hline White ethnicity & $1799(90)$ & $5845(96)$ & 21305 (95) & $72(94)$ & $18932(94)$ & $1322(96)$ \\
\hline Body mass index & $23.5(21.2-25.8)$ & $23.2(21.4-26.1)$ & $23.3(21.4-25.8)$ & $24.3(21.5-27.4)$ & $23.4(21.5-25.8)$ & $23.0(20.9-26.1)$ \\
\hline Physical activity, METs/wk & $9.1(2.9-21.7)$ & $6.5(2.4-16.5)$ & $9.3(3.2-21.0)$ & $7.1(1.9-17.4)$ & $8.8(20.9-3.0)$ & $6.1(2.1-15.9)$ \\
\hline Current smokers & $484(24)$ & $1520(25)$ & $4763(21)$ & $22(29)$ & $4514(23)$ & $434(32)$ \\
\hline Family history of hypertension & $786(40)$ & $2525(42)$ & $9078(41)$ & $30(39)$ & $8008(40)$ & $625(46)$ \\
\hline \multicolumn{7}{|l|}{ Nurses' Health Study II } \\
\hline Age, years & $40(35-45)$ & $44(39-49)$ & $44(39-50)$ & $40(36-45)$ & $43(38-49)$ & $43(38-48)$ \\
\hline White ethnicity & $2865(83)$ & $4821(96)$ & $20288(94)$ & $580(93)$ & $18231(93)$ & $2941(94)$ \\
\hline Body mass index & $23.0(21.0-26.2)$ & $24.7(21.9-28.9)$ & $23.3(21.1-26.5)$ & $26.1(22.6-31.8)$ & $23.4(21.3-26.9)$ & $24.0(21.5-28.3)$ \\
\hline Physical activity, METs/wk & $14.2(4.8-31.5)$ & $12.0(4.5-26.3)$ & $17.5(7.4-34.7)$ & $7.7(2.3-19.6)$ & $15.9(6.4-33.1)$ & $22.4(9.9-41.5)$ \\
\hline Current smokers & $392(10)$ & $696(11)$ & $2218(8)$ & $125(15)$ & $2157(9)$ & $516(13)$ \\
\hline Family history of hypertension & $1652(48)$ & $2578(49)$ & $10955(49)$ & $334(50)$ & $10035(49)$ & $1597(48)$ \\
\hline \multicolumn{7}{|c|}{ Health Professionals Follow-up Study } \\
\hline Age, years & $53(45-61)$ & $55(47-63)$ & $56(48-64)$ & $45(41-53)$ & $57(49-64)$ & $46(42-55)$ \\
\hline White ethnicity & $1212(82)$ & $3208(94)$ & $11076(91)$ & $365(92)$ & $11089(91)$ & $1266(93)$ \\
\hline Body mass index & $24.8(23.2-26.6)$ & $24.4(23.0-26.5)$ & $24.4(23.0-26.4)$ & $25.1(23.5-27.3)$ & $24.7(23.1-26.5)$ & $24.4(22.9-26.2)$ \\
\hline Physical activity, METs/wk & $12.6(3.3-30.1)$ & $12.1(4.1-29.6)$ & $15.3(5.0-33.4)$ & $7.5(1.9-20.1)$ & $13.9(4.5-31.3)$ & $10.4(3.1-27.1)$ \\
\hline Current smokers & $143(10)$ & $323(9)$ & $870(7)$ & $68(17)$ & $1013(8)$ & $149(11)$ \\
\hline Family history of hypertension & $450(30)$ & $1016(30)$ & $3742(31)$ & $128(32)$ & $2690(33)$ & $475(35)$ \\
\hline
\end{tabular}


(1.25, 1.11 to 1.41; P for trend<0.001) but not in HPFS $(0.95,0.84$ to 1.08; $P$ for trend $=0.46)$. The pooled hazard ratio comparing extreme categories in all three cohorts was 1.11 (0.96 to 1.28) in a random effect model and 1.11 (1.04 to 1.19) in a fixed effect model (table 3).

Increasing consumption of French fries ( $\geq 4$ servings/ week compared with $<1$ serving/month) was associated with an increased risk of hypertension in all three cohorts (table 4), with multivariable hazard ratios of 1.17 (0.92 to 1.50) in NHS, 1.17 (1.04 to 1.33) in NHS II, and 1.16 (1.00 to 1.33) in HPFS. The pooled hazard ratio was 1.17 (1.07 to 1.27; P for trend <0.001) in both random and fixed effect models. Potato chips were not associated with an increased risk of hypertension in NHS and NHS II; in HPFS, however, participants consuming four or more servings a week of potato chips had a lower risk of developing hypertension (hazard ratio $0.86,0.79$ to 0.93 ; $\mathrm{P}$ for trend=0.004) (table 5). Also, increased consumption of potato chips was associated with an increased risk of hypertension in NHS II (hazard ratio 1.24, 1.16 to 1.32; $P$ for trend $<0.001$ ) when we used an age adjusted model but not when we used a multivariate model (1.02, 0.96 to 1.09; $P$ for trend=0.61) (table 5). This loss of significance was primarily due to confounding by body mass index.

In the younger women (NHS II), the hypothetical fraction of new onset hypertension attributable to the consumption of more than one serving a month of total potatoes was $12.8 \%$, suggesting that this proportion of hypertension incidence might have been prevented had these young women (about 13/100) not consumed baked, boiled, or mashed potatoes or French fries.

Adjusting for sodium intake and other micronutrients, such as potassium, magnesium, calcium, and fiber, did not materially alter our results. Removing body mass index and

Table 2 | Pooled hazard ratios $(95 \% \mathrm{Cl})$ for incident hypertension for total consumption of potatoes (baked, boiled, or mashed potatoes and French fries) in Nurses' Health Study, Nurses' Health Study II, and Health Professional Follow-up Study

\begin{tabular}{|c|c|c|c|c|c|c|c|}
\hline & \multicolumn{5}{|c|}{ Consumption levels } & \multirow{2}{*}{$\begin{array}{l}\text { Linear } P \\
\text { for trend }\end{array}$} & \multirow{2}{*}{$\begin{array}{l}\text { P for } \\
\text { heterogeneit }\end{array}$} \\
\hline & $\leq 1$ per month & $1-3$ per month & 1-3 per week & 4-6 per week & $\geq 1$ per day & & \\
\hline \multicolumn{8}{|l|}{ Nurses' Health Study } \\
\hline Cases/person years & $291 / 10480$ & 4527/143881 & $18286 / 516243$ & $12085 / 344911$ & $539 / 18743$ & & \\
\hline Multivariate adjusted HR† & 1.00 (reference) & 1.00 (0.89 to 1.13$)$ & 1.05 (0.93 to 1.18$)$ & 1.09 (0.97 to 1.23$)$ & $1.07(0.92$ to 1.24$)$ & $<0.001$ & \\
\hline \multicolumn{8}{|l|}{ Nurses' Health Study II } \\
\hline Cases/person years & 156/13108 & $2065 / 127696$ & $13635 / 752459$ & $8947 / 430729$ & $443 / 20484$ & & \\
\hline Multivariate adjusted $\mathrm{HR} \dagger$ & 1.00 & 1.04 (0.88 to 1.22$)$ & $1.13(0.96$ to 1.32$)$ & 1.23 (1.04 to 1.44$)$ & $1.42(1.18$ to 1.72$)$ & $<0.001$ & \\
\hline \multicolumn{8}{|c|}{ Health Professionals Follow-up Study } \\
\hline Cases/person years & $156 / 6004$ & $1918 / 67267$ & $7958 / 261776$ & $6304 / 209045$ & $416 / 16138$ & & \\
\hline Multivariate adjusted HR† & 1.00 & 0.96 (0.81 to 1.13$)$ & 0.99 (0.84 to 1.16) & 1.01 (0.86 to 1.19$)$ & $0.96(0.79$ to 1.16$)$ & 0.20 & \\
\hline \multicolumn{8}{|l|}{ Pooled results } \\
\hline HR with random effect model & 1.00 & 1.00 (0.92 to 1.09$)$ & 1.05 (0.97 to 1.14$)$ & $1.11(1.02$ to 1.22$)$ & 1.13 (0.91 to 1.40$)$ & 0.02 & $<0.001$ \\
\hline HR with fixed effect model & 1.00 & $1.00(0.92$ to 1.09$)$ & $1.05(0.97$ to 1.14$)$ & $1.10(1.02$ to 1.20$)$ & $1.12(1.02$ to 1.24$)$ & $<0.001$ & \\
\hline
\end{tabular}

*Follow-up from 1984 to 2012

tHazard ration adjusted for age, race/ethnicity (white, African-American, Asian, Hispanic, other), body mass index, current smoking status, physical activity, weight change per food frequency questionnaire cycle, menopausal status (in NHS and NHS II), alcohol intake, current oral contraceptive use (in NHS II), analgesic use (non-steroidal anti-inflammatory drugs, acetaminophen (paracetamol), aspirin), family history of hypertension, total energy intake, animal flesh intake (combination of processed and unprocessed red meat, poultry, and seafood), whole grains (fifths), sugar sweetened drink intake, artificially sweetened diet drink intake, total fruit, total vegetables.

łFollow-up from 1991 to 2011

§Follow-up from 1986 to 2012

Table 3 | Pooled hazard ratios $(95 \% \mathrm{Cl})$ for incident hypertension for consumption of potatoes (baked, boiled, or mashed) in Nurses' Health Study, Nurses' Health Study II, and Health Professional Follow-up Study

\begin{tabular}{|c|c|c|c|c|c|c|}
\hline & \multicolumn{4}{|c|}{ Consumption levels } & \multirow{2}{*}{$\begin{array}{l}\text { P for } \\
\text { trend }\end{array}$} & \multirow{2}{*}{$\begin{array}{l}\text { P for } \\
\text { heterogeneity }\end{array}$} \\
\hline & $\leq 1$ per month & 1-3 per month & 1-3 per week & $\geq 4$ per week & & \\
\hline \multicolumn{7}{|l|}{ NHS } \\
\hline Cases/person years & $406 / 14882$ & $6233 / 189737$ & $22353 / 648475$ & $6736 / 181163$ & & \\
\hline Multivariate adjusted $\mathrm{HR} \dagger$ & 1.00 (reference) & 1.07 (0.97 to 1.19) & $1.12(1.01$ to 1.24$)$ & $1.13(1.02$ to 1.26$)$ & 0.01 & \\
\hline \multicolumn{7}{|l|}{ NHS II } \\
\hline Cases/person years & $333 / 25,721$ & $5673 / 327099$ & $16338 / 867668$ & $2902 / 123987$ & & \\
\hline Multivariate adjusted HR† & 1.00 & 1.08 (0.97 to 1.21) & 1.17 (1.04 to 1.31$)$ & 1.25 (1.11 to 1.41$)$ & $<0.001$ & \\
\hline \multicolumn{7}{|l|}{ HPFS } \\
\hline Cases/person years & $308 / 11050$ & $3088 / 102773$ & $10668 / 361971$ & $2688 / 84435$ & & \\
\hline Multivariate adjusted HRt & 1.00 & $0.92(0.82$ to 1.04$)$ & 0.95 (0.84 to 1.07) & 0.95 (0.84 to 1.08$)$ & 0.46 & \\
\hline \multicolumn{7}{|l|}{ Pooled results } \\
\hline HR with random effect model & 1.00 & 1.03 (0.93 to 1.13$)$ & 1.08 (0.96 to 1.21) & 1.11 (0.96 to 1.28$)$ & 0.05 & 0.002 \\
\hline HR with fixed effect model & 1.00 & 1.03 (0.97 to 1.10) & 1.08 (1.02 to 1.15$)$ & 1.11 (1.04 to 1.19) & $<0.001$ & \\
\hline
\end{tabular}

*Follow-up from 1984 to 2012

tHazard ration adjusted for age, race/ethnicity (white, African-American, Asian, Hispanic, other), body mass index, current smoking status, physical activity, weight change per food frequency questionnaire cycle, menopausal status (in NHS and NHS II), alcohol intake, current oral contraceptive use (in NHS II), analgesic use (non-steroidal anti-inflammatory drugs, acetaminophen (paracetamol), aspirin), family history of hypertension, total energy intake, animal flesh intake (combination of processed and unprocessed red meat, poultry, and seafood), whole grains (fifths), sugar sweetened drink intake, artificially sweetened diet drink intake, total fruit, total vegetables.

fFollow-up from 1991 to 2011

§Follow-up from 1986 to 2012 
Table 4 | Pooled hazard ratios $(95 \% \mathrm{Cl})$ for incident hypertension for consumption of French fries in Nurses' Health Study, Nurses' Health Study II, and Health Professional Follow-up Study

\begin{tabular}{|c|c|c|c|c|c|c|}
\hline & \multicolumn{4}{|c|}{ Consumption levels } & \multirow{2}{*}{$\begin{array}{l}\text { P for } \\
\text { trend }\end{array}$} & \multirow{2}{*}{$\begin{array}{l}\text { P for } \\
\text { heterogeneity }\end{array}$} \\
\hline & $\leq 1$ per month & 1-3 per month & 1-3 per week & $\geq 4$ per week & & \\
\hline \multicolumn{7}{|l|}{ NHS } \\
\hline Cases/person years & $14507 / 420566$ & $17912 / 506132$ & $3243 / 105756$ & $66 / 1802$ & & \\
\hline Multivariate adjusted $\mathrm{HR} \dagger$ & 1.00 (reference) & $1.08(1.06$ to 1.11$)$ & $1.11(1.06$ to 1.16$)$ & $1.17(0.92$ to 1.50$)$ & $<0.001$ & \\
\hline \multicolumn{7}{|l|}{ NHS II } \\
\hline Cases/person years & $4735 / 291260$ & $13887 / 719673$ & $6321 / 322226$ & $303 / 11316$ & & \\
\hline Multivariate adjusted HR† & 1.00 & 1.04 (1.00 to 1.08$)$ & 1.12 (1.07 to 1.17$)$ & $1.17(1.04$ to 1.33$)$ & $<0.001$ & \\
\hline \multicolumn{7}{|l|}{ HPFS } \\
\hline Cases/person years & $4723 / 165247$ & $7994 / 256288$ & $3817 / 131730$ & $218 / 6964$ & & \\
\hline Multivariate adjusted HR† & 1.00 & 1.04 (1.00 to 1.08$)$ & $1.08(1.02$ to 1.14$)$ & $1.16(1.00$ to 1.33$)$ & 0.002 & \\
\hline \multicolumn{7}{|l|}{ Pooled results } \\
\hline HR with random effect model & 1.00 & 1.06 (1.03 to 1.09$)$ & 1.11 (1.08 to 1.13$)$ & 1.17 (1.07 to 1.27$)$ & $<0.001$ & 0.14 \\
\hline HR with fixed effect model & 1.00 & $1.06(1.04$ to 1.08$)$ & 1.11 (1.08 to 1.13$)$ & 1.17 (1.07 to 1.27$)$ & $<0.001$ & \\
\hline
\end{tabular}

*Follow-up from 1984 to 2012

tHazard ration adjusted for age, race/ethnicity (white, African-American, Asian, Hispanic, other), body mass index, current smoking status, physical activity, weight change per food frequency questionnaire cycle, menopausal status (in NHS and NHS II), alcohol intake, current oral contraceptive use (in NHS II), analgesic use (non-steroidal anti-inflammatory drugs, acetaminophen

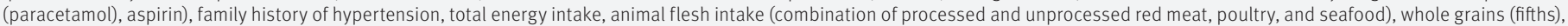
sugar sweetened drink intake, artificially sweetened diet drink intake, total fruit, total vegetables.

\#Follow-up from 1991 to 2011

§Follow-up from 1986 to 2012

Table 5 | Pooled hazard ratios $(95 \% \mathrm{Cl})$ for incident hypertension for consumption of potato chips in Nurses' Health Study, Nurses' Health Study II, and Health Professional Follow-up Study

\begin{tabular}{|c|c|c|c|c|c|c|}
\hline & \multicolumn{4}{|c|}{ Consumption levels } & \multirow{2}{*}{$\begin{array}{l}\text { P for } \\
\text { trend }\end{array}$} & \multirow{2}{*}{$\begin{array}{l}\text { P for } \\
\text { heterogeneity }\end{array}$} \\
\hline & $\leq 1$ per month & 1-3 per month & 1-3 per week & $\geq 4$ per week & & \\
\hline \multicolumn{7}{|l|}{ NHS } \\
\hline Cases/person years & $10704 / 309729$ & $15556 / 443075$ & $8310 / 245781$ & $1158 / 35672$ & & \\
\hline Multivariate adjusted $\mathrm{HR} \dagger$ & 1.00 (reference) & $1.02(0.99$ to 1.04$)$ & 1.07 (1.03 to 1.10$)$ & $1.03(0.97$ to 1.10$)$ & 0.004 & \\
\hline \multicolumn{7}{|l|}{ NHS II } \\
\hline Cases/person years & $3988 / 242232$ & $11367 / 581659$ & $8612 / 457599$ & $1279 / 62985$ & & \\
\hline Multivariate adjusted HR† & 1.00 & 1.05 (1.01 to 1.08$)$ & 1.05 (1.01 to 1.09) & 1.02 (0.96 to 1.09$)$ & 0.61 & \\
\hline \multicolumn{7}{|l|}{ HPFS } \\
\hline Cases/person years & $4533 / 149730$ & $6730 / 218121$ & $4747 / 164415$ & $742 / 27964$ & & \\
\hline Multivariate adjusted HR† & 1.00 & $0.96(0.92$ to 0.99$)$ & 0.97 (0.93 to 1.01) & $0.86(0.79$ to 0.93$)$ & 0.004 & \\
\hline \multicolumn{7}{|l|}{ Pooled results } \\
\hline HR with random effect model & 1.00 & 1.01 (0.96 to 1.05) & 1.03 (0.97 to 1.09) & 0.97 (0.87 to 1.08) & 0.98 & $<0.001$ \\
\hline HR with fixed effect model & 1.00 & 1.01 (0.99 to 1.03) & 1.04 (1.02 to 1.06$)$ & 0.98 (0.95 to 1.02) & 0.47 & \\
\hline
\end{tabular}

*Follow-up from 1984 to 2012

tHazard ration adjusted for age, race/ethnicity (white, African-American, Asian, Hispanic, other), body mass index, current smoking status, physical activity, weight change per food frequency questionnaire cycle, menopausal status (in NHS and NHS II), alcohol intake, current oral contraceptive use (in NHS II), analgesic use (non-steroidal anti-inflammatory drugs, acetaminophen (paracetamol), aspirin), family history of hypertension, total energy intake, animal flesh intake (combination of processed and unprocessed red meat, poultry, and seafood), whole grains

(fifths), sugar sweetened drink intake, artificially sweetened diet drink intake, total fruit, total vegetables.

łFollow-up from 1991 to 2011

§Follow-up from 1986 to 2012

weight change from the multivariable models did not change our results, and nor did adding trans, saturated, and polyunsaturated fat. We found no consistent interactions between any type of potato intake and either body mass index or age. Using simple updating rather than cumulative averaging to analyze the association of potato intake with hypertension yielded similar findings. Pooled hazard ratios did not materially change when we used random effect models compared with fixed effect models.

Table 6 reports substitution analyses. Replacing one serving a day of baked, boiled, or mashed potatoes with one serving a day of a non-starchy vegetable was associated with a lower adjusted pooled hazard ratio of 0.93 (0.89 to 0.96; $\mathrm{P}<0.001$ ).

\section{Discussion}

In three prospective cohorts of US women and men, we found that higher long term intake of baked, boiled, or mashed potatoes was significantly associated with an increased risk of hypertension in women, independent of numerous other predictors of risk of hypertension including dietary factors such as whole grain intake and whole fruit and vegetable intake. In addition, higher consumption of French fries was associated with incident hypertension in all three cohorts, whereas potato chip intake was associated with no increased risk. To our knowledge, this study is the first to examine potato consumption and the incidence of hypertension.

The association of potato intake with hypertension is a critical public health problem in the United States, in large part because potatoes have recently been inserted into government sponsored food programs. ${ }^{6}$ The justification for their inclusion into food programs is the potential cardiovascular benefits generated by their high potassium content, which in turn is primarily justified by beneficial effects on blood pressure. Specifically, the 


\begin{tabular}{|c|c|c|c|c|}
\hline Starchy vegetables & 0.99 (0.91 to 1.07) & 0.90 (0.81 to 0.99) & 1.05 (0.94 to 1.16$)$ & 0.98 (0.93 to 1.03); $P=0.42$ \\
\hline Non-starchy vegetables & $0.95(0.90$ to 1.00$)$ & $0.82(0.76$ to 0.88$)$ & 1.01 (0.94 to 1.09) & 0.93 (0.89 to 0.96); $P<0.001$ \\
\hline \multicolumn{5}{|c|}{$\begin{array}{l}\text { *Adjusted for age, race/ethnicity (white, African-American, Asian, Hispanic, other), body mass index, current smoking status, physical activity, weight } \\
\text { change per food frequency questionnaire cycle, menopausal status (in NHS and NHS II), alcohol intake, current oral contraceptive use (in NHS II), } \\
\text { analgesic use (non-steroidal anti-inflammatory drugs, acetaminophen (paracetamol), aspirin), family history of hypertension, total energy intake, animal } \\
\text { flesh intake (combination of processed and unprocessed red meat, poultry, and seafood), whole grains (fifths), sugar sweetened drink intake, artificially } \\
\text { sweetened diet drink intake, total fruit, total vegetables. } \\
\text { †Pooled hazard ratios for three cohorts from fixed effects model. }\end{array}$} \\
\hline
\end{tabular}

Institute of Medicine and the US Department of Agriculture recommended white potatoes to be allowed as part of the fruit and vegetable cash voucher WIC program, and most states have already implemented this regulation. The Institute of Medicine's report emphasized potatoes' high potassium content, which is a desired feature of foods for the WIC population, ${ }^{6}$ and high potassium intake has been associated with a lower blood pressure. In a meta-analysis of 22 randomized controlled trials, for example, increased potassium intake (as a supplement) produced a $3.5 \mathrm{~mm} \mathrm{Hg}$ reduction in systolic blood pressure and $2.0 \mathrm{~mm} \mathrm{Hg}$ reduction in diastolic blood pressure in people with hypertension. ${ }^{8}$ However, we found that higher potato intake was associated with an increased, rather than decreased, risk of developing hypertension.

\section{Potential mechanisms}

One potential mechanism that could explain our findings is the high glycemic load associated with potatoes. Boiled white potatoes have a high glycemic load of $21 .{ }^{20}$ Glycemic load is a measurement that reflects how a serving of a specific food affects glucose concentrations in the human body. It is obtained by multiplying the grams of carbohydrates in a food by the glycemic index of the food, divided by 100 (glycemic index compares $50 \mathrm{~g}$ of a specific food with $50 \mathrm{~g}$ of glucose and is reported on a scale of $0-100$, with higher scores given to food with the most rapid rise in glucose). ${ }^{2122}$ The postprandial hyperglycemia that follows a high glycemic load meal has been associated with endothelial dysfunction, oxidative stress, and inflammation, all potentially important mechanisms in the development of hypertension. ${ }^{23-25}$ The potential adverse consequence of a high carbohydrate intake, and therefore a higher glycemic load, on blood pressure in pre-hypertensive and hypertensive people was examined in the OMNIHEART trial. ${ }^{9}$ Compared with a carbohydrate rich diet, a protein rich diet and a diet rich in unsaturated fat lowered systolic and diastolic blood pressure by $1.4 / 3.5 \mathrm{~mm} \mathrm{Hg}$ and 1.3/2.9 mmHg, respectively, at six weeks.

Weight gain associated with potato intake is another potential mechanism for the development of hypertension. ${ }^{2627}$ As an example, in a prospective analysis of diet with weight change in the NHS, NHS II, and HPFS, each additional daily serving of potato chips and potatoes was strongly associated with four year weight change (1.7 and $1.3 \mathrm{lbs}$, respectively). ${ }^{28}$ However, we controlled for both weight change and body mass index in our multivariable models, reducing the likelihood that our findings are explained simply by changes in adiposity (we also removed weight change and body mass index from our models, and our results were mostly unchanged). Because excess adiposity is a strong risk factor for hypertension, our analyses that adjusted for adiposity likely underestimated the long term adverse effects of potatoes on risk of hypertension.

We found a few differences between the cohorts of women and men. An increased risk of hypertension with increasing consumption of boiled, baked, or mashed potatoes was seen in both female cohorts but not in the male cohort. These findings, as well as the lack of association of potato chips with the incidence of hypertension in HPFS, were unexpected. Whereas a non-significant trend toward a higher risk of developing hypertension was seen in women with increased consumption, men had a lower incidence of hypertension with greater long term intake of potato chips. Men who consumed more potato chips were these variables in our analysis. Most brands of potato chips are produced using monounsaturated and polyunsaturated fats instead of trans and saturated fats, which could be considered healthier. ${ }^{29}$ In a randomized double blind four week/three week crossover trial, for example, participants consuming potato chips fried in highly oleic sunflower oil (which is very high in monounsaturated fat) had reduced low density lipoprotein cholesterol compared with participants who consumed potato chips fried in palm olein, an oil high in saturated fats. ${ }^{30}$ However, controlling for polyunsaturated fat in secondary analyses did not eliminate the inverse association of potato chips with hypertension in men.

\section{Limitations of study}

This study has several limitations. Firstly, random misclassification of potato intake could have occurred because all dietary assessment methods are imperfect; however, this type of random error in assessment of exposure would tend to attenuate our findings and therefore underestimate the true association. Secondly, participants self reported a diagnosis of hypertension and direct blood pressure measurements were not obtained. However, our method of ascertainment of hypertension (self report by trained health professionals of similar educational backgrounds) has been extensively validated in all three cohorts. ${ }^{12-14}$ Thirdly, our population consists mostly of non-Hispanic white people of a relatively uniform socioeconomic status. However, we have no reason to suspect that the biological response to diet is qualitatively different across ethnic groups. Fourthly, as with any observational study, our findings could be explained by slightly younger and leaner, although we adjusted for 
residual confounding; for example, potatoes are often consumed with salt and added fat (such as butter or margarine). The increased sodium content could explain the association of boiled/baked potatoes with hypertension. However, our results did not materially change after we adjusted for intake of sodium or trans and saturated fat.

We also controlled for many known and proposed risk factors for hypertension that were collected in a prospective fashion. Furthermore, the relative uniformity of socioeconomic status reduces the likelihood of confounding by unmeasured variables.

\section{Conclusion}

We found independent prospective associations of higher intake of baked, boiled, or mashed potatoes and French fries with an increased risk of hypertension. These findings have potentially important public health ramifications, as they do not support a potential benefit from the inclusion of potatoes as vegetables in government food programs but instead support a harmful effect that is consistent with adverse effects of high carbohydrate intakes seen in controlled feeding studies.

We thank participants and staff of the Nurses' Health Study, the Nurses' Health Study II, and the Health Professionals Follow-up Study for their valuable contributions.

Contributors: LB and JFP designed this study. LB was involved in data collection. WCW, ERB, and JFP provided statistical expertise. LB analyzed the data and wrote the first draft of the manuscript. All authors helped with the interpretation of the results and approved the final version of the manuscript. LB is the guarantor.

Funding: This study was funded by research grants UM1 CA186107, R01 HL034594, UM1 CA176726, UM1 CA167552, and R01 HL35464 from the National Institutes of Health. LB was funded by an American Heart Association fellowship award (14POST20380070). The funding sources did not participate in the design of the study, the analysis or the interpretation of the data, the writing of this manuscript, or the process to submit this article for publication. The authors are not affiliated with the funding sources.

Competing interests: All authors have completed the ICMJE uniform disclosure form at www.icmje.org/coi_disclosure.pdf (available on request from the corresponding author) and declare: no support from any organization for the submitted work other than that described above; no financial relationships with any organizations that might have an interest in the submitted work in the previous three years; no other relationships or activities that could appear to have influenced the submitted work.

Ethical approval: The study protocol was approved by the institutional review boards of the Brigham and Women's Hospital and the Harvard School of Public Health. Participants provided implied consent by virtue of voluntarily returning their questionnaires.

Data sharing: No additional data available.

Transparency: The lead author confirms that the manuscript is an honest, accurate and transparent account of the study being reported; that no important aspects of the study have been omitted; and that any discrepancies from the study as planned (and, if relevant, registered) have been explained.

This is an Open Access article distributed in accordance with the Creative Commons Attribution Non Commercial (CC BY-NC 3.0) license, which permits others to distribute, remix, adapt, build upon this work non-commercially, and license their derivative works on different terms, provided the original work is properly cited and the use is noncommercial. See: http://creativecommons.org/licenses/by-nc/3.0/.

1 Summerbell CD, Waters E, Edmunds LD, Kelly S, Brown T, Campbell $\mathrm{KJ}$. Interventions for preventing obesity in children. Cochrane Database Syst Rev 2005·3:CD001871.

2 Kumanyika S, Jeffery RW, Morabia A, Ritenbaugh C, Antipatis VJ. Public Health Approaches to the Prevention of Obesity (PHAPO) Working Group of the International Obesity Task Force (IOTF). Obesity prevention: the case for action. Int J Obes Relat Metab Disord 2002;26:425-36. doi:10.1038/sj.ijo.0801938.

3 Healthy, Hunger-Free Kids Act of 2010. Pub. L. No. 111-296,124 Stat 3183, 2010

4 Institute of Medicine of the National Academies. WIC Food Packages Time for a Change. National Academies Press, 2006.
5 Food and Nutrition Service (FNS), USDA. Nutrition standards in the National School Lunch and School Breakfast Programs. Final rule. Fed Regist 2012;77:4088-167.

6 Rasmussen KM, Latulippe ME, Yaktine AL, eds. Review of WIC Food Packages: An Evaluation of White Potatoes in the Cash Value Voucher Letter Report. National Academies Press, 2015.

Diet, nutrition and the prevention of chronic diseases. World Health Organ Tech Rep Ser 2003;916:i-viii, 1-149, backcover.

8 Aburto NJ, Hanson S, Gutierrez H, Hooper L, Elliott P, Cappuccio FP. Effect of increased potassium intake on cardiovascular risk factors and disease: systematic review and meta-analyses. BMJ 2013;346:f1378. doi:10.1136/ bmj.f1378.

9 Appel LJ, Sacks FM Carey VJ, et al OmniHeart Collaborative Research Group. Effects of protein, monounsaturated fat, and carbohydrate intake on blood pressure and serum lipids: results of the OmniHeart randomized trial. JAMA 2005;294:2455-64. doi:10.1001/jama.294.19.2455.

10 Willett WC, Sampson L, Stampfer MJ, et al. Reproducibility and validity of a semiquantitative food frequency questionnaire. Am J Epidemiol 1985;122:51-65.

11 Rimm EB, Giovannucci EL, Stampfer MJ, Colditz GA, Litin LB, Willett WC Reproducibility and validity of an expanded self-administered semiquantitative food frequency questionnaire among male health professionals Am J Epidemiol 1992:135:1114-26, discussion 1127-36.

12 Colditz GA, Martin P, Stampfer MJ, et al. Validation of questionnaire information on risk factors and disease outcomes in a prospective cohort study of women. Am J Epidemiol 1986;123:894-900.

13 Forman IP, Curhan GC, Taylor EN. Plasma 25-hydroxyvitamin D levels and risk of incident hypertension among young women. Hypertension 2008;52:828-32. doi:10.1161/HYPERTENSIONAHA.108.117630.

14 Ascherio A, Rimm EB, Giovannucci EL, et al. A prospective study of nutritional factors and hypertension among US men. Circulation 1992;86:1475-84. doi:10.1161/01.CIR.86.5.1475.

15 Salvini S, Hunter DJ, Sampson L, et al. Food-based validation of a dietary questionnaire: the effects of week-to-week variation in food consumption. Int J Epidemiol 1989;18:858-67. doi:10.1093/ije/18.4.858.

16 Hu FB, Rimm E, Smith-Warner SA, et al. Reproducibility and validity of dietary patterns assessed with a food-frequency questionnaire. Am J Clin Nutr 1999:69:243-9.

17 Rimm EB, Stampfer MJ, Colditz GA, Chute CG, Litin LB Willett WC. Validity of self-reported waist and hip circumferences in men and women. Epidemiology 1990:1:466-73. doi:10.1097/00001648-199011000-00009.

18 Wolf AM, Hunter DJ, Colditz GA, et al. Reproducibility and validity of a self-administered physical activity questionnaire. Int J Epidemiol 1994:23:991-9. doi:10.1093/ije/23.5.991

19 Rockhill B, Newman B, Weinberg C. Use and misuse of population attributable fractions. Am J Public Health 1998;88:15-9. doi:10.2105/ AJPH.88.1.15.

20 Atkinson FS, Foster-Powell K, Brand-Miller JC. International tables of glycemic index and glycemic load values: 2008. Diabetes Care 2008:31:2281-3. doi:10.2337/dc08-1239.

21 Gagné $\mathrm{L}$. The glycemic index and glycemic load in clinical practice. Explore (NY) 2008;4:66-9. doi:10.1016/j.explore.2007.10.011.

22 Wolever TM, Jenkins DJ, Jenkins AL, Josse RG. The glycemic index methodology and clinical implications. Am J Clin Nutr 1991;54:846-54

23 Ludwig DS. The glycemic index: physiological mechanisms relating to obesity, diabetes, and cardiovascular disease. JAMA 2002;287:241423. doi:10.1001/jama.287.18.2414

24 Ceriello A, Bortolotti N, Crescentini A, et al. Antioxidant defences are reduced during the oral glucose tolerance test in normal and non-insulin-dependent diabetic subjects. Eur J Clin Invest 1998;28:329-33. doi:10.1046/j.1365-2362.1998.00295.x

25 Title LM, Cummings PM, Giddens K, Nassar BA. Oral glucose loading acutely attenuates endothelium-dependent vasodilation in healthy adults without diabetes: an effect prevented by vitamins $\mathrm{C}$ and $\mathrm{E}$. J Am Coll Cardiol 2000;36:2185-91. doi:10.1016/S0735-1097(00)00980-3.

26 Must A, Spadano J, Coakley EH, Field AE, Colditz G, Dietz WH. The disease burden associated with overweight and obesity. JAMA 1999;282:1523-9. doi:10.1001/jama.282.16.1523.

27 Hall JE. The kidney, hypertension, and obesity. Hypertension 2003:41:625-33 doi:10.1161/01.HYP.0000052314.95497.78.

28 Mozaffarian D, Hao T, Rimm EB, Willett WC, Hu FB. Changes in diet and lifestyle and long-term weight gain in women and men. N Engl J Med 2011;364:2392-404. doi:10.1056/NEJMoa1014296.

29 Gonçalves Albuquerque T, Sanches-Silva A, Santos L, Costa HS. An update on potato crisps contents of moisture, fat, salt and fatty acids (including trans-fatty acids) with special emphasis on new oils/fats used for frying. Int I Food Sci Nutr 2012;63:713-7. doi:10.3109/09637486.2011.644768.

30 Choudhury N, Truswell AS, McNeil Y. Comparison of plasma lipids and vitamin $\mathrm{E}$ in young and middle-aged subjects on potato crisps fried in palmolein and highly oleic sunflower oil. Ann Nutr Metab 1997:41:137-48. doi:10.1159/000177989. 